\title{
Grußwort des Rektors zum Sonderheft 90 Jahre Aufbereitung und Veredlung
}

\author{
Wilfried Eichlseder
}

Montanuniversität Leoben, Leoben, Österreich

Online publiziert 16. Oktober 2020

Sehr geehrte Leserinnen und Leser,

Das Fachgebiet der Aufbereitung leitet sich aus der Nutzbarmachung von mineralischen, in erster Linie bergmännisch gewonnenen Rohstoffen ab. Aufbereitungsverfahren zur weiteren Verarbeitung von Erzen, Kohlen, Salzen oder Industriemineralen dienen als Basis für die gesamte Produktionskette. Die zentrale Frage der zunehmenden Verknappung von Rohstoffen und deren Sicherstellung gewinnt weltweit zunehmend an Bedeutung. Damit geht die Erschließung neuer Lagerstätten und die effiziente Aufbereitung der abgebauten Rohstoffe einher.

Um der Bedeutung der Aufbereitung gerecht zu werden, wurde bereits 1930 an der Montanuniversität die Lehrkanzel für Aufbereitung und Veredlung eingerichtet. Deren Entwicklungsgeschichte beginnt mit O. Prof. Dr.-Ing. Ernst Bierbrauer, der aus der damaligen Erzabteilung des Kaiser-Wilhelm-Institutes für Eisenforschung in Düsseldorf an die Montanistische Hochschule berufen wurde und 37 Jahre lang in Forschung und Lehre wirkte. Dank seiner Tatkraft konnte trotz der damals schwierigen wirtschaftlichen Rahmenbedingungen innerhalb von zwei Jahren nach Institutsgründung eine voll arbeitsfähige Lehr- und Forschungseinrichtung aufgebaut werden, die auch zahlreiche Forschungsaufträge aus der Praxis erfolgreich bearbeitet hat. Über den langen Zeitraum von 1968 bis 2005, also ebenfalls 37 Jahre lang, leitete o. Univ. Prof. Dipl.Ing. Dr. mont. Hans Jörg Steiner die Geschicke des Institutes. Mit seinen Lehr- und Forschungsbestreben im Bereich der Aufbereitungstechnik war er weit über die Grenzen Österreichs hinaus ein anerkannter Fachmann und konnte zahlreiche Fragestellungen der Industrie im Bereich der Aufbereitung lösen. Univ.-Prof. Dipl.-Ing. Dr. mont. Helmut Flachberger, selbst ehemaliger Student von Prof. Steiner

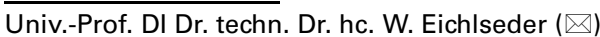

Montanuniversität Leoben,

Franz-Josef-Straße 18,

8700 Leoben, Österreich

wilfried.eichlseder@unileoben.ac.at

und angesehener Experte seines Fachbereichs, übernahm die Leitung des Lehrstuhls im Jahr 2005. Rückblickend betrachtet fielen in diesen Zeitraum seit 2005 u. a. die Planung, der Bau, die Besiedelung und die Inbetriebnahme eines Aufbereitungstechnikums und Aufbereitungslabors im Impulszentrum für Rohstoffe sowie der Aufbau des Schwerpunktfaches Aufbereitung und Veredlung im Masterstudium Rohstoffverarbeitung wie auch der Aufbau des Universitätslehrganges Rohstoffaufbereitung.

Die Forschungsaktivitäten rund um die Aufbereitung von Rohstoffen leiteten sich aus den vielfältigen Herausforderungen der Industriepartner ab. Die in diesem Zeitraum von 15 Jahren verfassten 28 Dissertationen legen eindrucksvoll Zeugnis von den vielfältigen Forschungsaktivitäten der Leobener Aufbereiterinnen und Aufbereitern ab.

Das vorliegende Sonderheft widmet sich dem 90-jährigen Bestehen des Lehrstuhls für Aufbereitung und Veredlung der Montanuniversität. Ich gratuliere herzlich zu diesem besonderen Jubiläum und wünsche für die Zukunft viele weitere Meilensteine im Bereich der Aufbereitung!

Mit freundlichem Glück auf! Wilfried Eichlseder

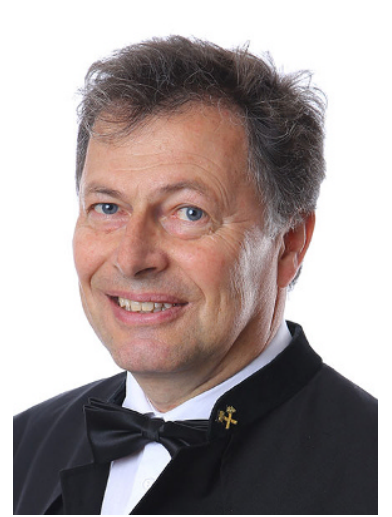

Wilfried Eichlseder 